Elsevier

HRR 01064

\title{
Potentiation of noise induced threshold shifts and hair cell loss by carbon monoxide
}

\author{
Laurence D. Fechter, John S. Young and Lynn Carlisle \\ Departments of Environmental Health Sciences and Otolaryngology Head and Neck Surgery, \\ Johns Hopkins Medical Institutions, Baltimore, Maryland, U.S.A. and Kresge Hearing Research Institute, \\ University of Michigan Medical School, Ann Arbor, Michigan, U.S.A.
}

(Received 21 September 1987; accepted 21 February 1988)

\begin{abstract}
Previous studies have determined that severe systemic hypoxia disrupts cochlear function acutely, but have suggested that augmentation of cochlear perfusion may successfully protect cochlear function under all but the most profound hypoxic treatments. In the current study we report on the chronic effects of simultaneous exposures to noise and carbon monoxide on pure tone thresholds and hair cell survival in rats. Following initial threshold determination, rats received acute exposure to carbon monoxide, noise, or both agents concurrently. Thresholds were evaluated 2-4 and 6-8 weeks later. The data show that carbon monoxide alone does not affect either auditory thresholds or compromise hair cells at the light microscopic level. The noise exposure alone produced variable, but quite limited permanent threshold shifts which were related to the power spectrum of the broad band noise that was employed. Hair cell loss was restricted to the basal turn of the cochlea. Simultaneous exposure to carbon monoxide and noise induced large threshold shifts at all frequencies studied, but the effect was greatest at the highest test frequency; an effect not consistent with the noise power spectrum. Widespread hair cell loss persisted over fully half of the basilar membrane in the most severely affected rat. Outer hair cells appear to be particularly vulnerable. Carbon monoxide plus noise did not appear to preferentially disrupt a particular row of outer hair cells. These data complement existing evidence that hyperoxia can mitigate against noise induced injury and reinforce the view that some types of noise induced damage may result from metabolic insufficiencies.
\end{abstract}

Carbon monoxide; Noise; Hypoxia; Ototoxicity; Cochlear metabolism; Synergistic ototoxicity

\section{Introduction}

The vulnerability of the cochlea to reduced oxygen delivery has most commonly been demonstrated in acute electrophysiological experiments. In these experiments, anoxia produced by a broad range of treatments including tracheal occlusion or 100\% nitrogen administration (Lawrence et al., 1975; Nuttall and Lawrence, 1980), and chemical asphyxiation with cyanide (Konishi and Kelsey, 1968) or carbon monoxide (CO) (Makishima et al., 1977) has been shown to disrupt both resting $\mathrm{AC}$ and $\mathrm{DC}$ potentials and to reduce compound action potential (CAP) amplitude and sharpness

Correspondence to: Laurence D. Fechter, Department of Environmental Health Sciences, Johns Hopkins School of Hygiene and Public Health, 615 N. Wolfe Street, Baltimore, MD 21205 , U.S.A. of hair cell tuning curves. Such effects have typically been observed under profound hypoxic conditions and the permanent consequences of such treatment, if any, have not been determined.

In contrast to such data showing cochlea dysfunction under life threatening hypoxia, a more recent experiment employing a range of acute $\mathrm{CO}$ exposures showed that the cochlea may be rather resistant to systematically induced hypoxia due to the large compensatory elevations in cochlear blood flow which accompany $\mathrm{CO}$ administration (Fechter et al., 1987) and which can be predicted to occur with other hypoxic treatments as well. In that study, disruption of the CAP occurred only following profound $\mathrm{CO}$ exposures. Repeated measurement of the CAP and cochlear microphonic (CM) showed that complete recovery was typically observed as carboxyhemoglobin levels declined to $35 \%$. It is interesting to note that the transient loss 
of the CAP occurred only at the highest frequencies studied as this suggests either differences in metabolic activity of elements subserving high frequency auditory function and presumably located in the basal turn of the cochlea or differential oxygen supply to different turns of the cochlea.

There is a body of data which suggests that hyperoxia as well as hypoxia may play an important role under conditions of noise exposure. Carbogen administration has been reported to increase perilymph $\mathrm{pO}_{2}$ and temporal artery bloodflow in humans (Fisch et al., 1976). It has also been reported to speed recovery from noiseinduced temporary threshold shifts (TTS) in humans and chinchillas (Joglekar et al., 1977) and decrease the extent of TTS when administered prior to noise exposure (Witter et al, 1980). Carbogen can reduce temporary changes in N1 (Brown et al., 1985) and permanent changes in AC cochlear potentials and hair cell loss (Brown et al., 1982). Dengerink et al. (1984) have suggested that carbogen increases cochlear blood flow and may counteract bloodflow decreases induced by noise.

On the other hand, $\mathrm{CO}$ present concurrently with broad band noise can lead to potentiation of permanent noise induced threshold shifts (Young et al., 1987); CO plus noise resulted in both quantitatively more auditory loss and also yielded a higher frequency auditory loss than did noise presented alone. Such findings complement the carbogen experiments and strengthen the view that noise can produce permanent threshold shifts via metabolic factors. However, only two test frequencies were studied in that work and so limited data are available concerning possible differences between basal and more apical elements in metabolic requirements. The current experiment extends that of Young et al. (1987) by studying the effects of $\mathrm{CO}$ and noise exposure over a wider portion of the rat's auditory range and by providing histopathological data obtained from surface preparations of the organ of Corti. Carbon monoxide binds to hemoglobin with an affinity approximately 200 times greater than oxygen. It not only reduces the oxygen carrying capacity of hemoglobin, but also impedes the dissociation of oxygen from oxyhemoglobin by producing a conformational change in hemoglobin. In the rat carboxyhemoglobin levels reach a steady state after approximately $60-90 \mathrm{~min}$ and complete $\mathrm{CO}$ elimination occurs by $8 \mathrm{~h}$ after exposure stops. As in the case of hypoxic hypoxia and hypercapnia, carbon monoxide also induces profound cardiovascular consequences. Transitory, dose related elevation in cerebral blood flow (Traystman and Fitzgerald, 1977) and cochlea blood flow (Fechter et al., 1987) has been shown to result from CO exposure.

\section{Methods}

\section{Subjects}

Subjects were 16 male Long-Evans hooded rats (Blue Spruce Farms), weighing between 300 and $350 \mathrm{~g}$ at the start of testing. Subjects were group housed in acrylic cages in a colony room with a $12: 12$ hour light: dark cycle (lights on at 0700 ), and an ambient temperature of $21 \pm 2^{\circ} \mathrm{C}$. All animals had free access to water and to rat chow. Ambient noise levels in the colony room were between 40 and $50 \mathrm{dBA}$.

\section{Apparatus}

The apparatus we employ for reflex modulation audiometry has been described in an earlier publication (Young and Fechter, 1983). Individual chambers for testing four animals simultaneously are contained within an IAC 1200 audiometric room. Within each chamber is a spring-mounted cage, to which a magnet is attached such that it passes through the center of a wire coil. Startle responses cause the magnet to move with the cage, inducing a voltage in the coil which is proportional to cage velocity, and hence, to the amplitude of the startle response. Separate audio systems are used to present startle-eliciting stimuli (white noise bursts of $20 \mathrm{~ms}$ duration at $105 \mathrm{dBA}$ ), and startle-modulating stimuli (prestimuli), which are pure-tone pips of $200 \mathrm{~ms}$ duration with $5 \mathrm{~ms}$ on- and off-ramps. All stimulus presentations and data recording are under the control of a dedicated microprocessor. Stimuli are calibrated with a Brüel \& Kjaer model 2610 measuring amplifier, using a $1 / 2$ inch condenser microphone for tones of $2.5-10 \mathrm{kHz}$ and a $1 / 4$ inch condenser microphone for higher frequency tones. Pure tone intensity is measured free field by placing the micro- 
phone at the approximate height of the subjects' ears and at $2.5 \mathrm{~cm}$ spacings around test cage. The variability in intensities for each tone is $5 \mathrm{~dB}$ or less across the test cage.

Acute exposures to noise and/or CO took place in an IAC sound attenuating chamber designed for small animal experiments. The dimensions of the animal chamber were $40 \mathrm{~cm}$ deep $\times 60 \mathrm{~cm}$ wide $\times 36 \mathrm{~cm}$ high. The interior surface of the chamber was stainless steel, providing a reverberant environment. Four piezoelectric tweeters in the upper corners of the chamber were driven by a laboratory-built noise-source, amplified by an SAE model 201 amplifier. Subjects were exposed singly, in a wire-mesh cage $(8 \mathrm{~cm} \times 17$ $\mathrm{cm} \times 19 \mathrm{~cm}$ ) in the center of the chamber. Sound levels were monitored with a Quest model 215 Type 2 sound level meter, with the microphone at the approximate level of the rats' ears. A 1/3-octave analysis of the noise in the chamber was performed using a Bruel \& Kjaer 2112 analyzer and a $1 / 2$ inch condenser microphone. The noise had maximum intensity between 4 and $8 \mathrm{kHz}$, with rolloff of approximately $16 \mathrm{~dB} /$ octave. The noise spectrum used has previously been published (Young et al., 1987).

\section{Procedures}

The application of reflex modulation procedures to the assessment of auditory function has been described in previous papers (Fechter and Young, 1983; Fechter et al., 1986; Young and Fechter, 1983). The method provides estimates of stimulus detection sensitivity which are comparable to those obtained with conditioning procedures (e.g. Kelly and Masterton, 1977). The method is sensitive to ototoxic damage (Young and Fechter, 1983, 1986; Fechter et al., 1986), and yet is robust in the face of nonauditory (neuromuscular) toxicity (Fechter and Young, 1983). Reflex modification audiometry is based upon the finding that low intensity pure tone stimuli inhibit the response to a subsequent reflex eliciting stimulus. The extent of reflex inhibition is directly related to intensity of the pure tone stimulus as it increases above a detection threshold. Although a white-noise burst is used to elicit the acoustic startle reflex, it is important to remember that estimates of auditory thresholds are based upon the inhibition of the reflex produced by low-intensity pure-tone prestimuli, rather than absolute reflex amplitude.

Auditory threshold estimates were obtained for nine tones between 2.5 and $40 \mathrm{kHz}$ in approximate half-octave steps. Each tone was presented at 19 intensities using a $5 \mathrm{~dB}$ step between intensities by the method of constant stimuli. The frequency-intensity combinations were randomized within a test block of 110 trials. Eight of these test blocks were presented each day.

Data are routinely discarded from the first day of testing to control for warmup effects (which were pronounced in earlier studies e.g. Young and Fechter, 1983); the median response amplitudes from days 2 to 14 for each prestimulus frequency were subjected to a square-root transformation and fit with a knotted spline function. This computer-generated curve fitting procedure takes into account the fact that the function relating reflex inhibition to tone frequency is somewhat sigmoidal rather than linear. This procedure allows the use of information from data collected at all prestimulus intensities to estimate the threshold of reflex inhibition. Auditory thresholds are defined as a point on the spline function corresponding to $15 \%$ inhibition of the startle reflex.

Auditory thresholds were re-assessed in all subjects between 2-4 weeks after exposures to noise and/or $\mathrm{CO}$. An additional test of thresholds was conducted in two subjects from each group 6-8 weeks after toxic exposures in order to address the issue of permanence of the threshold shifts observed.

Following initial threshold determination (on days 2-14), one subject from each squad of four animals was assigned to one of the experimental conditions: noise alone, noise plus $\mathrm{CO}, \mathrm{CO}$ alone, untreated control. For all subjects except those in the control group, exposures took place on a single day, immediately following the last day of the initial threshold determination.

Noise exposure was for $120 \mathrm{~min}$ at a level of 105 dBA. Carbon monoxide exposure was for 210 min at $1200 \mathrm{ppm}$ (for comparison, pure cigarette smoke contains 30000 to $50000 \mathrm{ppm} \mathrm{CO}$, and alveolar $\mathrm{CO}$ levels during smoking are about 300 ppm). For the combined exposure, $90 \mathrm{~min}$ of $\mathrm{CO}$ exposure preceded noise onset, so that carbo- 
xyhemoglobin levels would be substantially at equilibrium during the noise exposure. Control subjects remained in the colony room on the day on which the other subjects were exposed to noise, $\mathrm{CO}$, or noise plus $\mathrm{CO}$.

\section{Statistical analyses}

Analyses of variance were run to compare preexposure thresholds among all treatment groups and to compare post exposure thresholds among treatment groups. Within subject analyses of variance were also conducted in which pre- and postexposure auditory thresholds were compared at each test frequency for each subject as a function of type of experimental treatment.

\section{Histopathological examination}

At the completion of threshold testing cight weeks after toxicant and/or noise exposure, two animals from each treatment group were sacrificed, the organ of Corti examined under light microscopy and cytocochleograms produced. The subjects were anaesthetized with ketamine ( 87 $\mathrm{mg} / \mathrm{kg})$ and xylazine $(13 \mathrm{mg} / \mathrm{kg})$, sacrificed by decapitation and the auditory bullas harvested bilaterally. The round and oval windows were opened, a small hole was made in the apex of the bony wall of the cochlea and a solution of $2.5 \%$ glutaraldehyde and $2 \%$ paraformaldehyde in phosphate buffer was gently perfused through the cochlea. Following a 24-h fixation, the tissue was thoroughly rinsed and placed in phosphate buffer.

One cochlea from each animal was post fixed in osmium tetroxide for one hour, dehydrated through a series of ethyl alcohols (35-50-70\%; $3 \times 10 \mathrm{~min}$ ) and microdissected. Then the organ of Corti was mounted on glass slides in glycerol. The second cochlea from each animal was post fixed in osmium tetroxide, dehydrated to $70 \%$ ethanol and microdissected. The segments of organ of Corti were further dehydrated (80-90-95-100-100\% for $10 \mathrm{~min}$ each). After two changes of propylene oxide : epon (1:1 overnight, $1: 44 \mathrm{~h}$, full strength overnight) the segments were embedded in fresh epon in slide molds. The epon slides were cured in a 60 degree oven for two days.

Histological damage was determined by counting hair cells and making cytocochleograms which present percent remaining hair cells as a function of distance along the basilar membrane from the apical end of the cochlea. The slides were coded and the counting was done without knowledge of experimental condition.

\section{Results}

As in previous work using reflex modification audiometry, auditory thresholds prior to experimental treatment were equivalent in sensitivity to those previously reported for the rat (Kelly and Masterton, 1977) (See Table I). Auditory thresholds averaged between -4 and $15 \mathrm{~dB}$ SPL across the various tones used in this experiment and there were no differences in initial auditory thresholds among the four treatment groups ( $F=$ $2.13,0.10<P>0.05$ ). Figs. 1 and 2 present the effects of $\mathrm{CO}$ alone, noise alone and the two agents given in combination upon pure tone auditory thresholds as a shift from each subject's preexposure audiogram. As compared either to untreated control subjects or to their own preliminary audiometric test (see Fig. 1), subjects exposed to $\mathrm{CO}$ alone did not show any shift in auditory thresholds. Indeed, both untreated controls and the $\mathrm{CO}$ subjects tended to show slightly

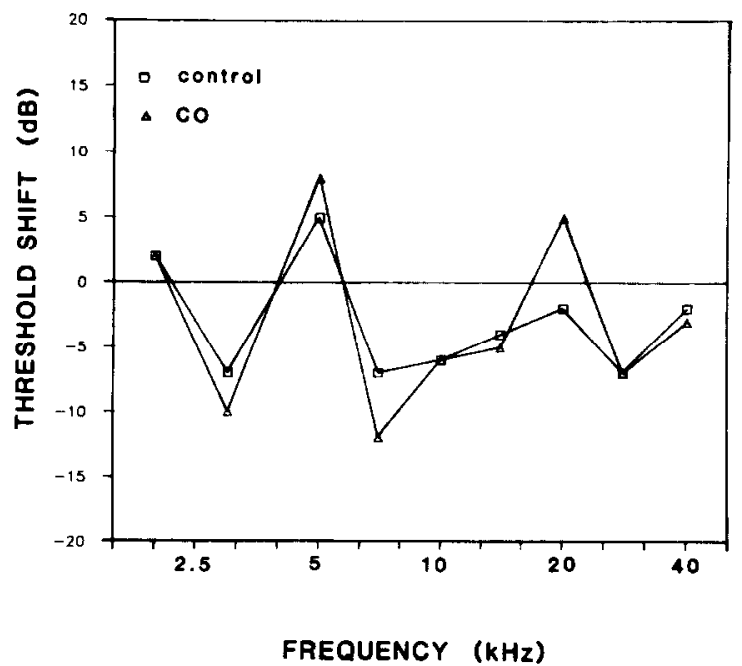

Fig. 1. Auditory threshold shift from pretreatment values (solid linc) measurcd between 2 and 4 weeks after an acute $1200 \mathrm{ppm}$ carbon monoxide exposure (open triangles) or no treatment (open squares). Loss of threshold sensitivity is indicated by a positive shift. There is no statistical evidence for a shift in threshold in either of these groups. 
TABLE I

PURE TONE THRESHOLDS FOR SUBJECTS PRIOR TO NOISE AND CARBON MONOXIDE EXPOSURE

\begin{tabular}{|c|c|c|c|c|c|c|c|c|c|}
\hline & \multicolumn{9}{|c|}{ Tone frequency $(\mathrm{kHz})$} \\
\hline & 2.5 & 3 & 5 & 7 & 10 & 14 & 20 & 28 & 40 \\
\hline $\bar{X}$ threshold dB SPL & 7.5 & 15 & 13 & 6 & -2 & 2 & 4 & -4 & -1 \\
\hline S.E. & 6 & 3 & 3 & 4 & 4 & 4 & 4 & 2 & 3 \\
\hline
\end{tabular}

better threshold sensitivity during the post-treatment auditory test than they had initially. This apparent improvement in thresholds upon repeated testing has been observed before (e.g. Young et al., 1987) and represents some change in the subjects' general behavior in the test situation which reduces variability of the test behavior. Subjects exposed to noise alone showed, as a group, rather minimal threshold shifts (see Fig. 2); the apparent shifts were generally less than $10 \mathrm{~dB}$ with respect to pre-treatment and tended to be greatest in the frequency range from $10-14 \mathrm{kHz}$ while $1 / 3$ octave band analysis showed that the noise had predominant energy between $4-8 \mathrm{kHz}$. An analysis of variance failed to find significant

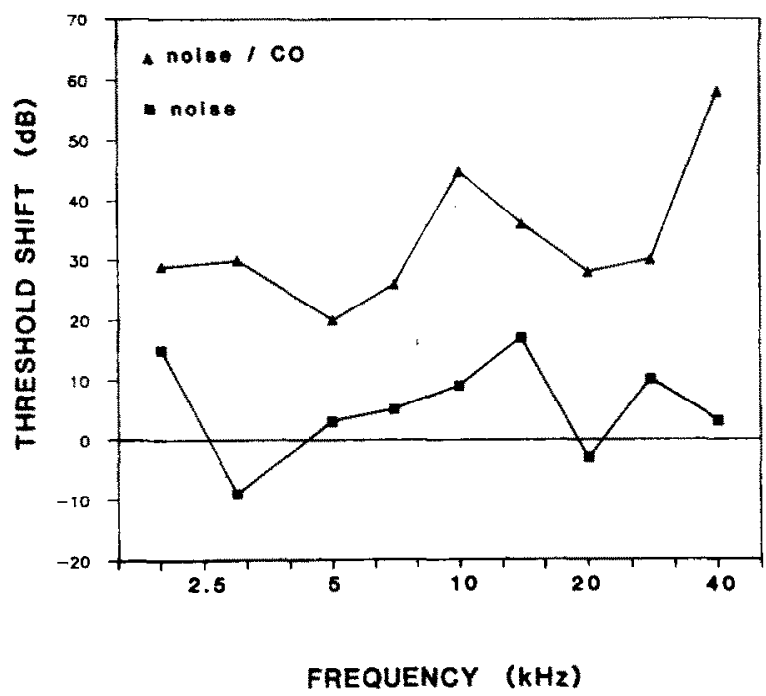

Fig. 2. Auditory threshold shift from pretreatment values (solid line) measured between 2 and 4 weeks following 110 dBA broad band noise (filled squares) or simultaneous noise and carbon monoxide (filled triangles). Loss of threshold sensitivity is indicated by a positive shift. Only the combined noise-carbon monoxide group shows statistically significant loss in threshold sensitivity relative to its own pre-exposure test. differences between pre- and post-noise exposure conditions $(F=1.64, P>0.20)$. By contrast with the $\mathrm{CO}$ subjects and the noise only group, those subjects exposed to both agents concurrently show a profound loss of auditory sensitivity following exposure (see Fig. 2). The most severe disruption in behaviorally determined threshold occurred at the highest test frequency, $40 \mathrm{kHz}$, and at $10 \mathrm{kHz}$.
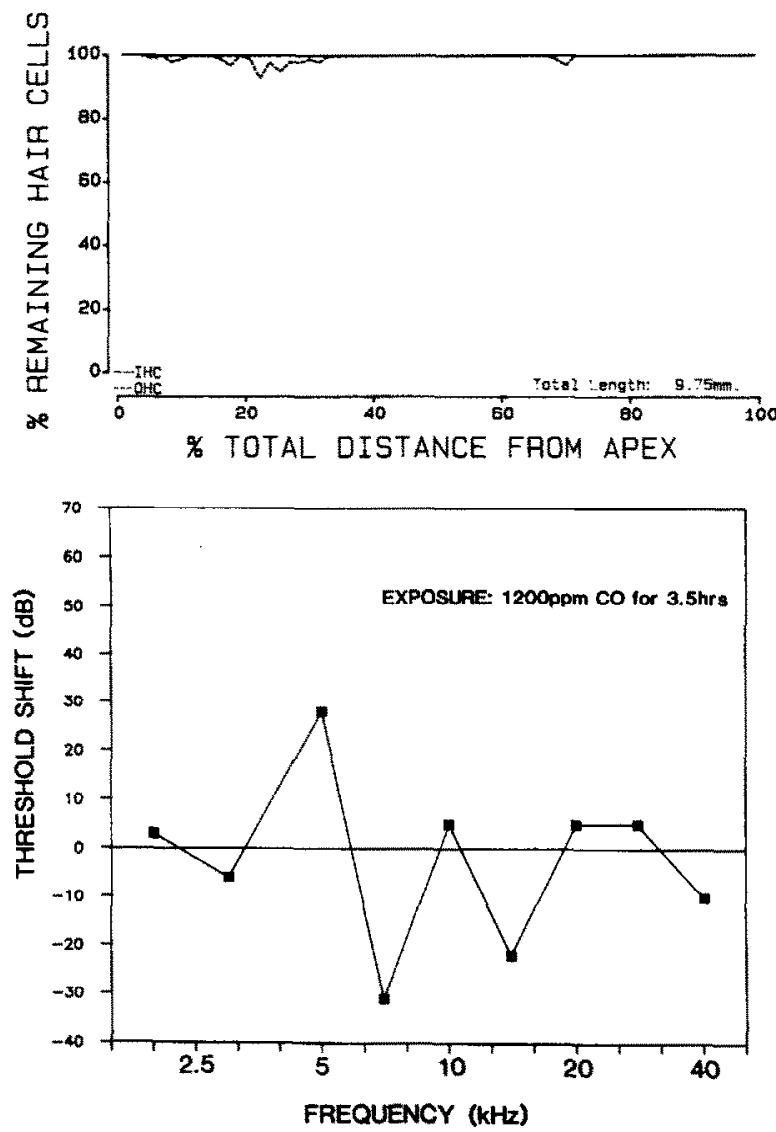

Fig. 3. Individual threshold shift data and subsequent cytocochleogram prepared from the organ of Corti of a subject treated with carbon monoxide acutely eight weeks earlier. 
That threshold loss was frequency related is supported by a significant tone $\mathrm{X}$ test session interaction $(F=3.77, \quad P<0.01)$. A Duncan's multiple range test did not provide evidence that the loss of threshold sensitivity at $10 \mathrm{kHz}$ actually represents more significant loss than observed between 14 and $28 \mathrm{kHz}$. There was no evidence of recovery of auditory sensitivity among the CO-noise exposed subjects tested between 6-8 weeks after exposure (data not shown).

The histopathological data obtained 8 weeks following exposure to the test agents were generally consistent with the behavioral findings. Carbon monoxide treatment alone did not produce a loss of hair cells (see Fig. 3). Although occasional outer hair cells (OHC) were missing, the amount of loss was within normal limits as can be seen by comparison with the cytocochleo-
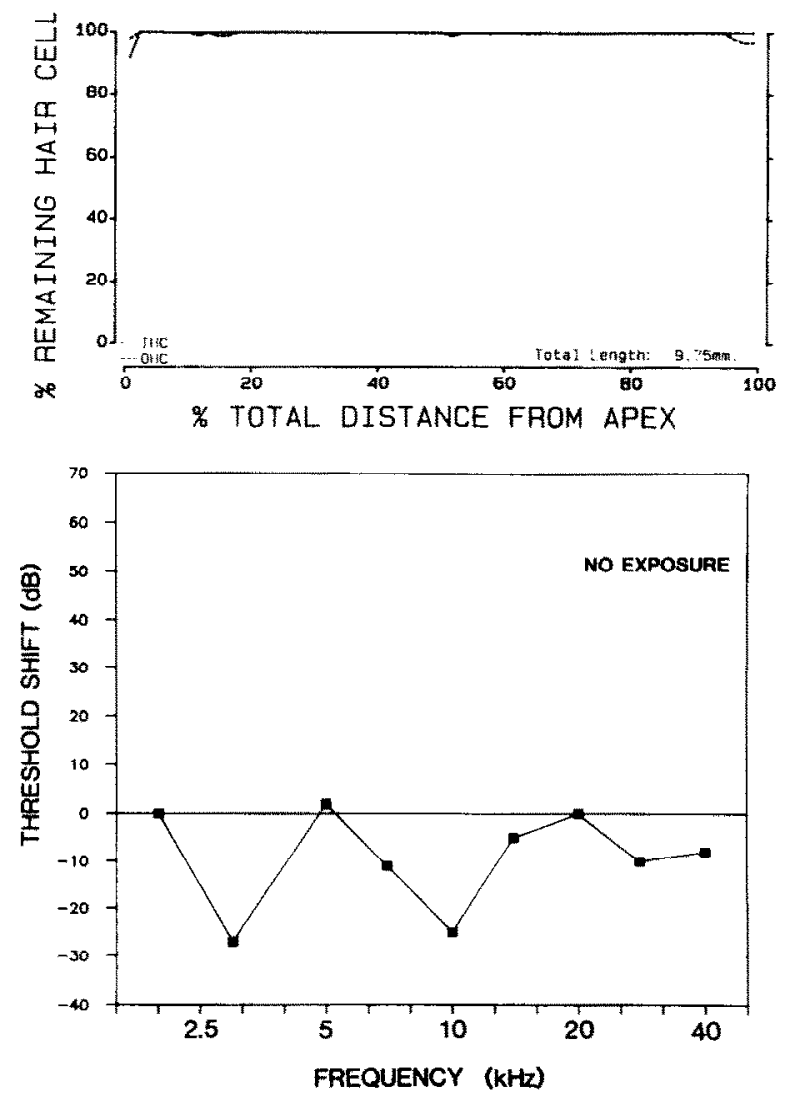

Fig. 4. Individual threshold shift data and subsequent cytocochleogram prepared from the organ of Corti of a non-exposed control subject which received auditory testing.
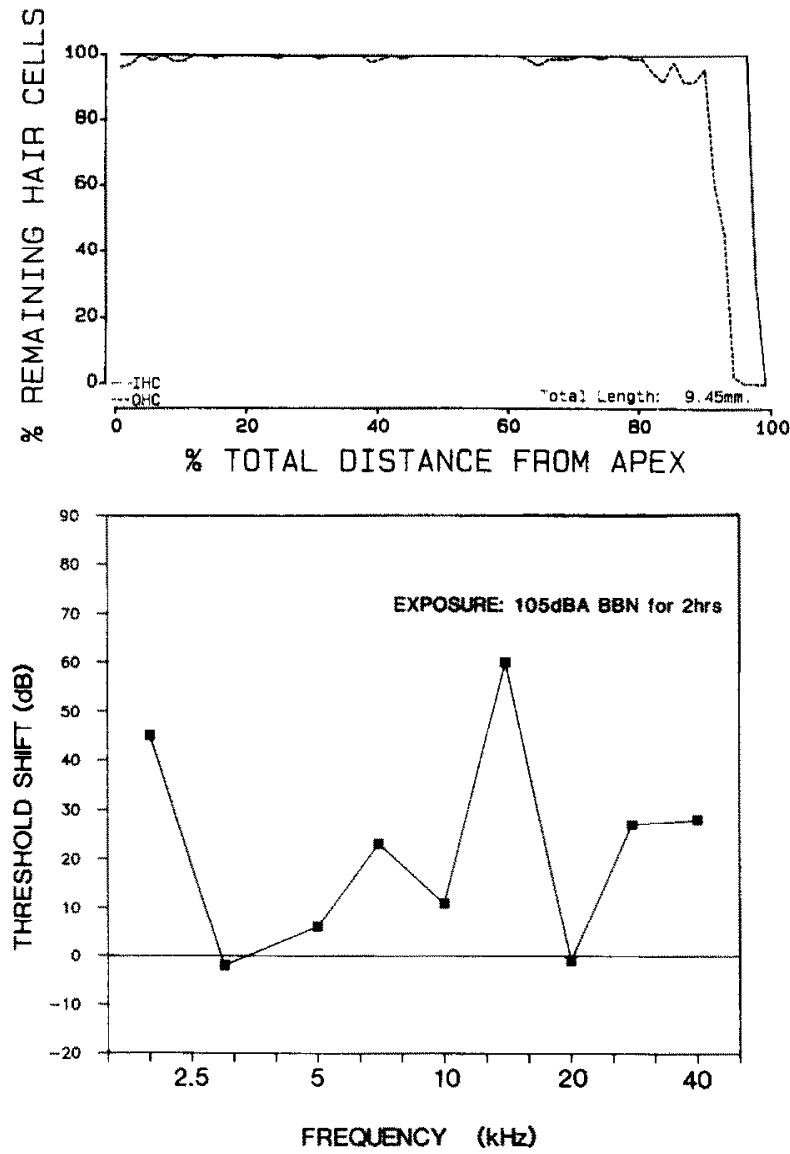

Fig. 5. Individual threshold shift data and subsequent cytocochleogram prepared from the organ of Corti of a subject treated with noise eight weeks earlier and showing the greatest. amount of auditory loss for this treatment group.

gram from the cochlea of a control subject (see Fig. 4). Noise exposure alone caused hair cell loss in the extreme basal portion of the organ of Corti (see Fig. 5); the particular animal whose cochlea is represented here showed the largest shift in auditory threshold of any animal in that treatment group and also the greatest amount of histopathology. For this subject we measured auditory threshold shifts of approximately $30 \mathrm{~dB}$ at 28 and $40 \mathrm{kHz}$ and a $60 \mathrm{~dB}$ loss at $14 \mathrm{kHz}$. Both $\mathrm{OHC}$ and inner hair cells (IHC) were missing in this subject, though the OHC loss was more extensive. Exposure to noise and $\mathrm{CO}$ resulted in a greater loss of sensory cells (see Fig. 6) than did noise alone. Damage consisted of complete loss of IHC extending approximately $15 \%$ of the distance from 

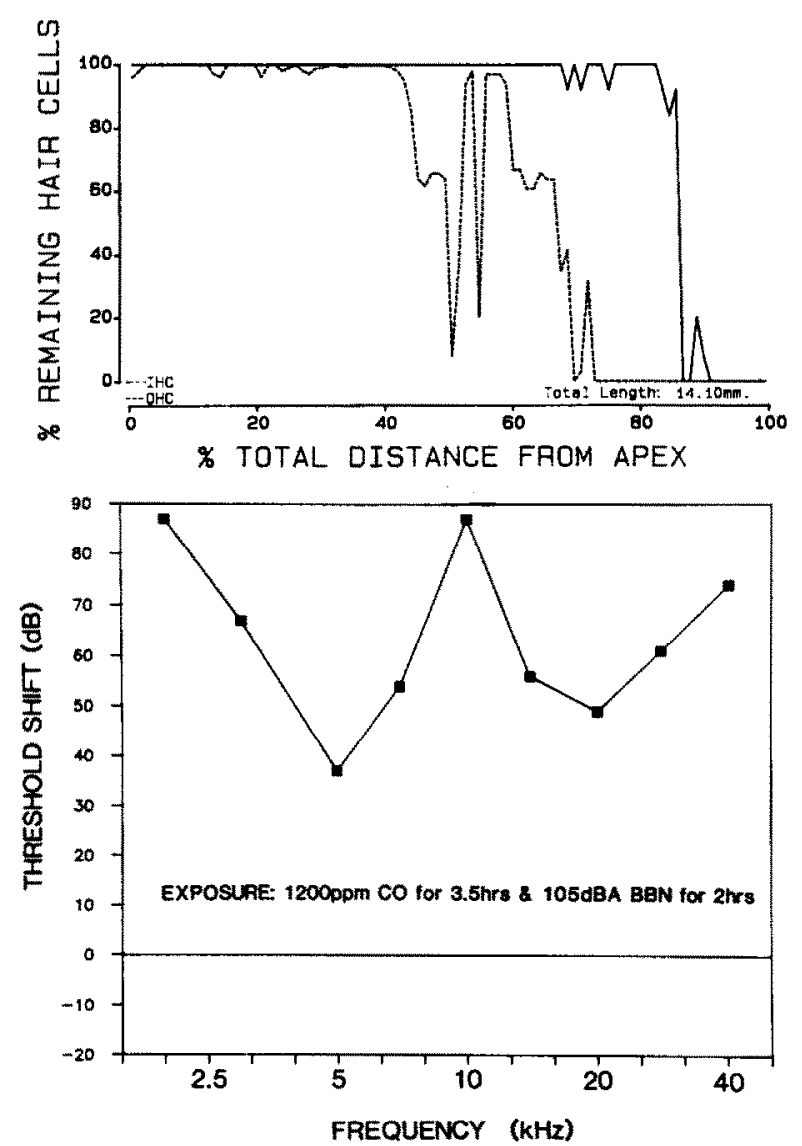

Fig. 6. Individual threshold shift data and subsequent cytocochleogram prepared from the organ of Corti of a subject treated simultaneously with noise and carbon monoxide eight weeks earlier and showing the greatest amount of auditory loss for this treatment group.

the basal end of the organ of Corti and loss of $\mathrm{OHC}$ extending up to $60 \%$ of the distance from the basal end of the organ of Corti. This subject showed the most profound shifts in behavioral thresholds of any subject within this treatment group and the other noise-CO treated subject showed histopathological changes more restricted to the basal turn with threshold shifts consistent with that locus of injury.

\section{Discussion}

The present work clearly demonstrates that acute $\mathrm{CO}$ intoxication can potentiate permanent noise induced threshold shifts and increase hair cell loss associated with that functional loss. This work bolsters a previous experiment (Young et al., 1987 ) in which we obtained evidence for potentiation of noise induced threshold shifts at the higher of two test frequencies ( $40 \mathrm{kHz}$ vs $10 \mathrm{kHz}$ ). The current data provide more extensive data relating to the frequency specificity of the effect. They show that simultaneous administration of noise and $\mathrm{CO}$ yields a profound loss of auditory threshold sensitivity at all test frequencies and particular vulnerability of thresholds for high frequency tones. Under the pattern of administration and testing used in this work, $\mathrm{CO}$ does not produce a threshold shift by itself.

The data also substantially strengthen the proposed mechanistic basis by which noise and hypoxia are thought to interact. While it previously had been shown that enhanced cochlear perfusion induced by carbogen gas exposure may reduce noise induced auditory dysfunction (e.g. Joglekar et al., 1977; Witter et al., 1980; Brown et al., 1982, 1984) we demonstrate that the converse, potentiation of noise induced injury by hypoxia, is also true. However, as opposed to previous hypoxia and hyperoxia-noise interaction studies, the present data demonstrate that permanent consequences of an acute hypoxic insult are possible if noise is present simultaneously. The histopathological data appear to show only quantitatively greater loss among the combined exposure group than in the noise exposed group rather than a qualitatively different pattern of damage. However, further histological study of the cochlear vasculature, for example, was not carried out and it cannot be concluded that the resulting injury produced by noise and noise plus $\mathrm{CO}$ does not show differences in kind as well as degree. The pattern of injury that was observed was to both IHC and OHC toward the basal turn with $\mathrm{OHC}$ more vulnerable than IHC. Carbon monoxide exposure alone did not result in permanent damage to hair cells at a light microscopic level. For comparison, electrophysiological studies in which acute hypoxic treatment or asphyxiation has been applied have suggested that both $\mathrm{IHC}$ and $\mathrm{OHC}$ may be affected. The CAP (Fechter et al., 1987) and tuning curve tip sensitivity (Russell and Cowley, 1983; Brown et al., 1983; Nuttall, 1984) are particularly disrupted by hypoxia with the cochlear 
microphonic also showing depression though generally to a lesser extent (Misrahy et al., 1958; Konishi and Kelsey, 1968; Brown et al., 1983; Fechter et al, 1987).

These data are important for several reasons. They have clinical and public health importance in that they establish that environmental pollutants may affect the vulnerability of organisms to noise-induced injury. Indeed, they show that two specific agents, noise and $\mathrm{CO}$, which did not produce any detectable threshold shift when given individually are able to produce profound auditory dysfunction following simultaneous exposure. Further dose response data using lower $\mathrm{CO}$ concentrations will be helpful in undertaking human risk assessment evaluations. Low level $\mathrm{CO}$ is ubiquitous in urban societies and is particularly associated with transportation, cigarette smoking and as a by-product of combustion. Thus, vehicular traffic, home heating, and industrial settings are important sources of exposure.

The results are also important to basic hearing science because of their relevance to questions of mechanisms of noise induced injury and of oxygen delivery and utilization by cochlear tissue. One interpretation of these data is that persisting noise exposure may act to enhance cochlear metabolism and that the simultaneous reduction in oxygen carrying capacity and delivery may render hair cells more vulnerable to permanent injury. The finding that hair cell injury occurs primarily in the basal turn and that high frequency auditory thresholds are particularly disrupted suggests either that cells in the basal turn have a higher rate of metabolic activity or poorer oxygen delivery. These data are consistent with the finding that acute $\mathrm{CO}$ exposure produces a transient loss in CAP sensitivity specifically for high frequency tones (Fechter et al., 1987).

\section{Acknowledgements}

This research was supported in part by NIHPHS grants No. ES02852 and RCDA No. ES 125 to LDF and by Center grant No. ES03819 to the Johns Hopkins University Department of Environmental Health Sciences. LC was supported in part by a NIH-PHS training grant No. NS07106 to the Kresge Hearing Research Institute at the
University of Michigan Medical Center. The authors gratefully acknowledge the technical assistance of Cindy Richard, Kevin Kaatz and Blair Miller in the conduct of this work. The authors are grateful to Dr. John Heinz of the John F. Kennedy Institute for the use of sound survey equipment and to Brenda Klamut for preparation of this manuscript for publication.

\section{References}

Brown, J.J., Vernon, J.A. and Fenwick, J.A. (1982) Reduction of acoustically induced auditory impairment by inhalation of carbogen gas. I. Permanent noise-induced cochlear damage. Acta Otolaryngol. 93, 312-328.

Brown, M.C., Nuttall, A.L., Masta, R.I. and Lawrence, M. (1983) Cochlear inner hair cells: Effects of transient asphyxia on intracellular potentials. Hear. Res. 9, 131-144.

Brown, J.J., Meikle, M.B. and Lee, C.A. (1985) Reduction of acoustically induced auditory impairment by inhalation of carbogen gas. Acta Otolaryngol. 100, 218-228.

Dengerink, H., Axelsson, A., Miller, J. and Wright, W. (1984) The effect of noise and carbogen on cochlear vasculature. Acta Otolaryngol. 98, 81-88.

Fechter, L.D. and Young, J.S. (1983) Discrimination of auditory from nonauditory toxicity by reflex modulation audiometry: effects of triethyltin. Toxicol. Appl. Pharmacol. 70, 216-227.

Fechter, L.D., Young, J.S. and Nuttall, A.L. (1986) Trimethyltin ototoxicity: evidence for a cochlear site of injury. Hear. Res, 23, 275-282.

Fechter, L.D., Thorne, P.R. and Nuttall, A.L. (1987) Effects of carbon monoxide and cochlear electrophysiology and blood flow. Hear. Res. 27, 37-45.

Fisch, U., Murata, K. and Hossli, G. (1976) Measurement of oxygen tension in human perilymph. Acta Otolaryngol. 81, $278-282$

Joglekar, S.S., Lipscomb, D.M. and Shambaugh, G.E. (1977) Effects of oxygen inhalation on noise-induced threshold shifts in humans and chinchillas. Arch. Otolaryngol. 103, $574-578$.

Kclly, J. and Masterton, B. (1977) Auditory sensitivity of the albino rat. J. Comp. Physiol. Psychol. 91, 930-936.

Konishi, T. and Kelsey, E. (1968) Effect of cyanide on cochlear potentials. Acta Oto-Laryngol. 65, 381-399.

Lawrence, M., Nuttall, A. and Burgio, P. (1975) Cochlear potentials and oxygen associated with hypoxia. Ann. Otol. Rhinol. Laryngol. 84, 499-51.

Makishima, K., Keane, W.M., Vernose, G.U. and Snow, J.B. (1977) Hearing loss of a central type secondary to carbon monoxide poisoning. Trans. Am. Acad. Ophthal. Otolaryngol. 84, 452-457.

Misrahy, G.A., Shinabarger, E.W. and Arnold, J.E. (1958) Changes in cochlear endolymphatic oxygen availability, action potential, and microphonics during and following asphyxia, hypoxia, and exposure to loud sounds. J. Acoust. Soc. Am. 30, 159-162. 
Nuttall, A.L. (1984) Dynamic aspects of guinea pig inner hair cell receptor potentials with transient asphyxia. Hear. Res. $16,1-16$.

Nuttall, A. and Lawrence, M. (1980) Endocochlear potential and scala media oxygen tension during partial anoxia. Am. J. Otolaryngol. 1, 147-153.

Russell, I.J. and Cowley, E. (1983) The influence of transient asphyxia on receptor potentials in inner hair cells of the guinea pig cochlea. Hear. Res. 11, 373-384.

Traystman, R.J. and Fitzgerald, R.S. (1977) Cerebral circulatory responses to hypoxic hypoxia and carbon monoxide hypoxia in carotid baroreceptor and chemoreceptor denervated dogs. In: D.H. Ingvar and N.A. Lassen (Eds.), Cerebral Function, Metabolism and Circulation, Munksgaard, Copenhagen, Denmark, pp. 294-295.
Witter, H.L., Deka, R.C., Lipscomb, D.M. and Shambaugh, G.E. (1980) Effects of prestimulatory carbogen inhalation on noise-induced temporary threshold shifts in humans and chinchilla. Am. J. Otol. 1, 227-232.

Young, J. and Fechter, L. (1983) Reflex inhibition procedures for animal audiometry: A technique for assessing ototoxicity. J. Acoust. Soc. Am. 73, 1686-1693.

Young, J. and Fechter, L. (1986) Trimethyltin exposure produces an unusual form of toxic auditory damage in rats. Toxicol. Appl. Pharmacol. 82, 87-93.

Young, J.S., Upchurch, M., Kaufman, M. and Fechter, L.D. (1987) Carbon monoxide exposure potentiates highfrequency auditory threshold shifts induced by noise. Hear. Res. 26, 37-43. 